\title{
On the modeling of aircraft tire
}

\author{
A. Kongo Kondéa,b, I. Rosu ${ }^{b}$, F.Lebon ${ }^{b}$, O.Brardo ${ }^{a}$, B.Devésa ${ }^{a}$ \\ a AIRBUS, 316 route de Bayonne, 31060 Toulouse cedex 03, France \\ b LMA-CNRS, Aix-Marseille Univ., 31 chemin Joseph-Aiguier, 13402 Marseille cedex 20, France
}

\begin{abstract}
A method is presented here for modeling and predicting the rolling and yaw behavior of an aircraft tire which is subjected to a strong inflation pressure and a concentrated load on the axle, in contact with a flat, rigid surface. Finite element methods were used to model and simulate the aircraft tire/ground interactions. The incompressibility of the material, the large transformations and the unilateral contact with Coulomb friction law were all taken into account. Imaging methods were used to examine the complex structure of the tire cross-section. Comparisons are made between the data obtained with the model, the experimental data and those provided by the manufacturer. The tire response predictions were found to depend considerably on the material and the geometrical characteristics of the tire.
\end{abstract}

\section{Introduction}

Modeling the geometry of tires and predicting their behavior are complex tasks. The loading, which can be either quasi-static or dynamic, also involves severe contact conditions.

\subsection{Review of the literature}

Most studies on tires have been performed on automobile tires [4]. Several authors $[9,4,13,15]$ have focused on truck tires, especially those used on military trucks, and the motion of vehicles has been simulated on various types of soil (dry, wet, muddy), whereas some authors have dealt with bike tires [17].

In the aerospatial and aeronautical field, the first studies on these lines were conducted by NASA $[16,11,3]$. These studies were mostly experimental and dealt with the thermal aspects (the influence of the temperature on tires dynamic responses, its distribution within the tire thickness, and the evolution of the friction coefficient in tire/ground contact).

In recent studies [14], numerical tools have been used to simulate the behavior of aircraft tires, and some modeling aspects still cause engineers and researchers major problems because there are too many non-linear phenomena involved.

Under operating conditions, tire's behavior is highly non-linear due to their constituents, their geometry and shape, the loading conditions, contact with friction and many other interconnected parameters.

\footnotetext{
* Corresponding author.

E-mail address: rosu@lma.cnrs-mrs.fr (I. Rosu).
}

The operating conditions and the structure of aircraft tires are very different from those of automobiles. For example, automobile tires are inflated at a pressure of 2 bars, whereas the nominal inflation pressure of aircraft tires is around 15 bars. The vertical loads are also extremely different: 1 to 6 tonnes in the case of automobiles and trucks, as compared with about 20 tonnes in that of aircraft [14].

Developing a fine, efficient mesh is difficult and requires lots of resources and it is necessary to simplify the geometry of numerical models (the grooves, asperities, etc.). The challenge is how to find a compromise between material models, geometrical models and the quality of the expected results. Finite Element Analysis (FEA) is now being used routinely to analyze and predict the various aspects of tire behavior. For these predictions to be successful, accurate 3-D tire models are essential. Another fundamental requirement for tire-modeling is the need for accurate, relevant information about the tire cross-section, especially as regards the layout of the composite structure.

\subsection{Aims of the study}

In this study, a numerical model for a smooth tire (without any grooves) is developed for static and dynamic aircraft tire simulations. A method of accurately modeling aircraft tires is presented. Based on experiments performed on samples taken from each of the layers in the tire, three models are developed: an orthotropic elastic model, an isotropic hyper elastic model and a composite model involving embedded elements called the "Rebar" model [1]. The mesh is obtained by rotating the axisymmetric 2-D section about the axle of the rim. The grooves are not included in this geometric model, for reasons shown previously in [6]. The grooves 


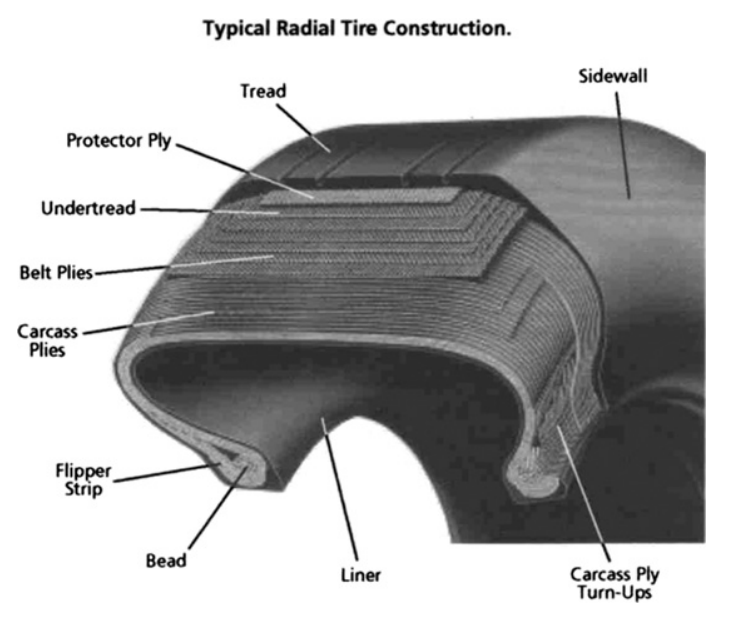

Fig. 1. Aircraft tires components.

are not essential because this study focused on the overall behavior of the tire.

Sensitivity studies will be performed in order to determine the influence of geometrical and material parameters on the response of the tire. For reasons of confidentiality, the figures presented here will show only general numerical results.

\section{Tire simulation using finite element methods}

In this study, finite element analyses were performed in several stages. In the first stage, the tire was modeled under inflation pressure using an axisymmetric model 2-D. A 3-D model was then developed for performing static vertical loading simulations (i.e. in the footprint stage). To study the lateral, torsional and longitudinal stiffness of the tire, a quasi-static analysis was performed after the static analysis. The thermal effects due to contact with friction were not taken into account in this study.

\subsection{Tire structure}

Modern pneumatic tires consist of a specific combination of rubber compounds, cord and steel belts (see Fig. 1). The main parts of a modern pneumatic tire are its body, sidewalls, beads, and tread. The body is made of rubberized fabric layers called plies, that give the tire its strength and flexibility. The fabric used is rayon, nylon, or polyester cord. The sidewalls and tread are made of chemically treated rubber. Embedded in the two inner edges of the tire are steel loops called beads, supporting the rim of the tire. The rubber components have different characteristics depending on their functional role. The tread, for example, comes into direct contact with the ground and has to be much harder than the sidewalls.

\subsection{Tire geometry}

Some imaging methods developed for inspection purposes are now being used by researchers to acquire the geometric data when no CAD tire geometry data are available. These methods include those based on charge-coupled devices (CDD), cameras, X-ray tomography devices and laser holography equipment, on both the micro and macro scales.

Scanned images give an accurate description of the perimeter of tire cross-section and the locations of the ply lines and cord ends.

The tire cross-section was cut in this study using a water-jet, as shown in Fig. 2. This method gives a highly accurate cutting plane and a very detailed image of all inner layers of the tire, as shown in Fig. 3.

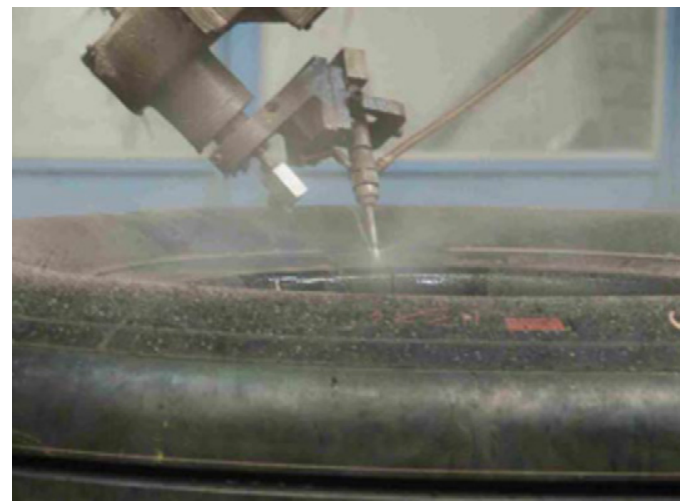

Fig. 2. Water-cutting process.

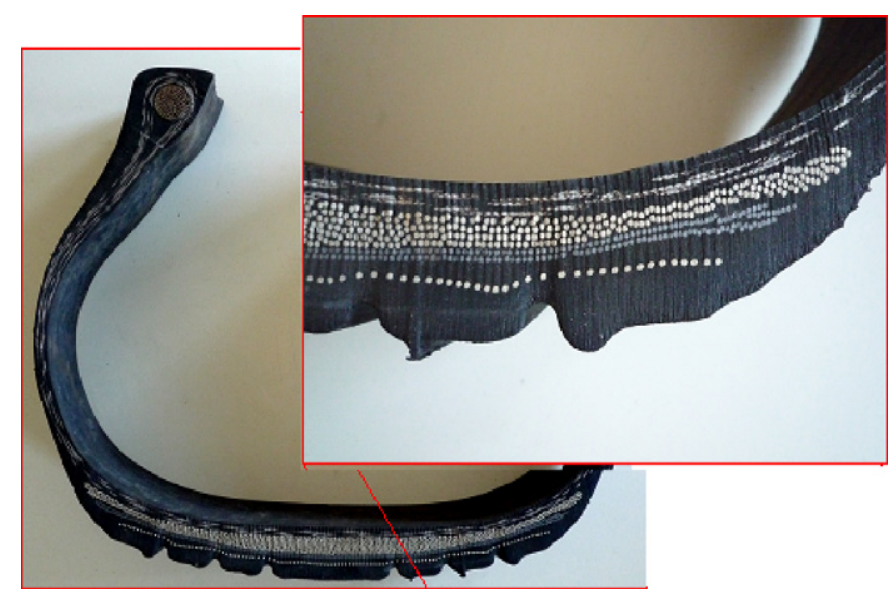

Fig. 3. Layout of the composite structure.

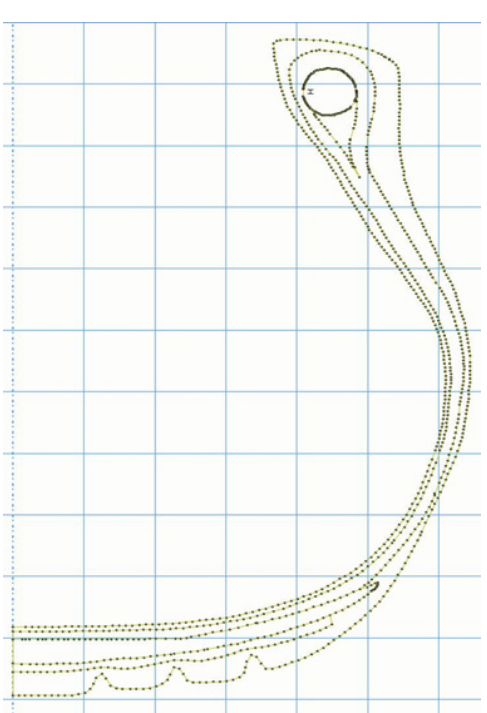

Fig. 4. Discretized tire structure.

Image processing methods, were used to discretize the crosssectional image is order to obtain the real 2-D tire structure, as shown in Fig. 4.

\subsection{Constitutive models for tire materials}

Since rubber is a highly extensible material, small-strain elasticity theory is not suitable for describing the responses of tires to large strains. A useful means of measuring the response consists 
in using the mechanical energy $W$ stored in the unit volume by deformation.

In tire applications, rubber compounds are often assumed to be isotropic, and cord/rubber composites are assumed to be orthotropic. These components combined can withstand the structural and thermal working conditions to which tires are exposed. In this section we will briefly describe the hyperelastic models and the linear orthotropic properties used so far to define the mechanical characteristics of the rubber, the belt and the ply layers. In this study, the material behavior will not be taken to depend on the temperature, as established experimentally in [7]. ${ }^{1}$

Orthotropic elastic properties have often been used in numerical modeling studies based on methods such as FEA and closedform methods. Since a tire undergoes large deformations, the overall structural problem is non-linear. However, the stiffness predictions obtained in the framework of linear orthotropic elasticity are useful for design purposes. In addition, when these predictions are combined with non-linear FEA methods, a good approximation of the tire's structural response is obtained. The model developed here for composite lamina and laminates was based on those presented in [5] and [18].

\subsubsection{Hyperelastic constitutive models for rubbers}

In tire modeling studies, the incompressible Mooney-Rivlin model is commonly and widely used $[4,8,14,15,20]$.

The stresses for hyperelastic materials can be obtained from the partial derivatives of the strain energy functions [10]. It is well known that in the case of pure stress state like uniaxial tension, biaxial tension and planar tension, the stress can simply be described in terms of stretch ratios.

Under uniaxial tensile stress conditions, we have:

$\lambda_{1}=\lambda, \quad \lambda_{2}=\lambda_{3}=\lambda^{-\frac{1}{2}}, \quad \lambda=1+\varepsilon$

where $\lambda_{i}$ are the principal stretch ratios, $\lambda$ is the stretch ratio and $\varepsilon$ is the strain.

The two first deviatoric strain invariants are

$\bar{I}_{1}=\lambda^{2}+2 \lambda^{-1}, \quad \bar{I}_{2}=\lambda^{-2}+2 \lambda$

The principle of virtual work and the material incompressibility $(D=\infty)$ are used to obtain the nominal stress-strain relationship,

$\delta U=T \delta \lambda=\frac{\partial U}{\partial \lambda} \delta \lambda$

and it follows that

$$
\begin{aligned}
T & =\frac{\partial U}{\partial \lambda}=\frac{\partial U}{\partial \bar{I}_{1}} \cdot \frac{\partial \bar{I}_{1}}{\partial \lambda}+\frac{\partial U}{\partial \bar{I}_{2}} \cdot \frac{\partial \bar{I}_{2}}{\partial \lambda} \\
& =2\left(1-\lambda^{-3}\right)\left(\lambda \frac{\partial U}{\partial \bar{I}_{1}}+\frac{\partial U}{\partial \bar{I}_{2}}\right)
\end{aligned}
$$

where $U$ is the strain energy function of incompressible hyperelastic materials and $T$ is the stress. Based on experimental data, the coefficients of the constitutive function can be determined for each rubber by performing curve fitting.

Since the Mooney-Rivlin model is widely used in tire modeling studies, this model will be compared with Neo-Hookean and Yeoh models in terms of their predictive power and their accuracy. The criterion on which the choice of models was based was the deformation rate reached (35 to 50\%) in aircraft applications [12].

The strain energy used in the three models is:

\footnotetext{
1 In this experiment, it was established that the temperature variations are restricted to the tread zone.
}

Neo-Hookean: $\quad U=C_{10}\left(\bar{I}_{1}-3\right)$

Mooney-Rivlin: $\quad U=C_{10}\left(\bar{I}_{1}-3\right)+C_{01}\left(\bar{I}_{2}-3\right)$

Yeoh: $\quad U=C_{10}+C_{20}\left(\bar{I}_{1}-3\right)^{1}+C_{30}\left(\bar{I}_{1}-3\right)^{2}$

As regards the predictive validity of these models, we used only the uniaxial tension test data to identify all the coefficients by performing curve fitting before comparing the three models. The results are presented in the next section.

\subsubsection{Mechanical behavior of composite cord/rubber materials}

Composite materials consist of at least two different constituents or components that are bonded together, giving a structure that meets specific thermo-mechanical requirements. The composite materials used for structural applications often include either continuous or chopped fibers embedded in a softer matrix.

The geometric complexity of tires makes it difficult to cut samples for performing classical experimental tests. Samples were therefore cut here in the radial and circumferential directions, but not in that of the thickness.

The reinforcement of a tire can be modeled in three ways:

1. Orthotropic elastic properties (0): When reinforced rubber is modeled using the orthotropic elastic approach, the reinforcement (steel cords, nylon cords, etc.) is taken to be a homogeneous component. This means cord/rubber composites such as belts and carcass and bead reinforcements are modeled in the form of orthotropic elastic components for which all the elastic modules are determined by performing tension tests on various samples in the radial and/or circumferential directions. The shear modulus and the elastic modulus in the thickness are computed using numerical samples, and the Poisson coefficients are determined by applying the following stability conditions ((10) and (11))

$$
\left[\begin{array}{l}
\epsilon_{1} \\
\epsilon_{2} \\
\epsilon_{3} \\
\gamma_{1} \\
\gamma_{2} \\
\gamma_{3}
\end{array}\right]=\left(\begin{array}{cccccc}
\frac{1}{E_{1}} & \frac{-v_{21}}{E_{2}} & \frac{-v_{31}}{E_{3}} & 0 & 0 & 0 \\
\frac{-v_{12}}{E_{1}} & \frac{1}{E_{2}} & \frac{-v_{32}}{E_{3}} & 0 & 0 & 0 \\
\frac{-v_{13}}{E_{1}} & \frac{-v_{23}}{E_{2}} & \frac{1}{E_{3}} & 0 & 0 & 0 \\
0 & 0 & 0 & \frac{1}{G_{12}} & 0 & 0 \\
0 & 0 & 0 & 0 & \frac{1}{G_{23}} & 0 \\
0 & 0 & 0 & 0 & 0 & \frac{1}{G_{31}}
\end{array}\right)\left[\begin{array}{c}
\sigma_{1} \\
\sigma_{2} \\
\sigma_{3} \\
\tau_{1} \\
\tau_{2} \\
\tau_{3}
\end{array}\right]
$$

The stability of an orthotropic material also requires that [S] must be positive-definite, which results in the criteria:

$$
\begin{aligned}
& E_{1}, E_{2}, E_{3}, G_{12}, G_{23}, G_{31}>0 \\
& \left|v_{i j}\right|<\left(\frac{E_{i}}{E_{j}}\right)^{\frac{1}{2}} i, j=1,2,3 \\
& 1-v_{12} v_{13}-v_{13} v_{23}-v_{12} v_{23}-2 v_{12} v_{13} v_{23}<0
\end{aligned}
$$

2. Isotropic Hyperelastic model $(H)$ : Reinforced rubber is modeled using the hyperelastic Yeoh model, and by performing simple tension tests on radially and circumferentially reinforced specimens.

3. Rebar approach $(R)$ : With the Rebar approach, the reinforcement is treated like a one-dimensional element [1] (see Fig. 5). The Rebar properties depend on the elastic modulus, which is determined performing a simple tension test. The viscosity of the rubber is not taken into account. The rubber is assumed to be purely hyperelastic.

The properties of Rebar components differ from those of the underlying component, and their orientation can be defined relative to the local coordinate system as shown in Fig. 5. To 
define the Rebar reinforcement, one must specify the crosssectional area $A$ of each Rebar component, the spacing $S$ between two consecutive cords, and the orientation angle $\theta$ of the Rebar cord in the local frame.

\subsubsection{Experimental determination of the properties of rubber}

To determine the properties of the tire material studied, uniaxial tension tests were performed on each rubber material, the steel beads and the reinforcement cords. Fig. 6 shows the specimens used in the tire rubber tests.

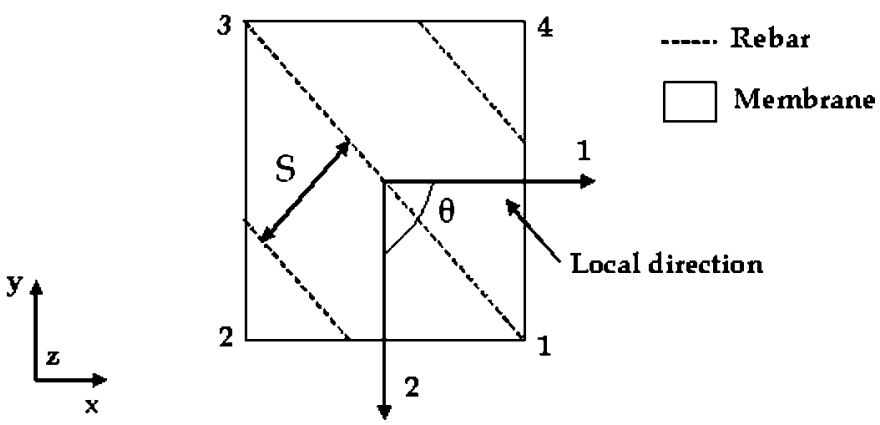

Fig. 5. Rebar components defined relative to the local coordinate system.

\subsection{Experimental results on tire specimens}

Uniaxial tension tests were carried out with an INSTRON 10KN Testing Machine with a large deformation extensometer. Fig. 7 gives the stress-strain curve obtained on the tread rubber in several tests.

Comparisons between the various predictions obtained on tread rubber materials are made in Fig. 8. These comparisons show that:

- The Mooney-Rivlin and Neo-Hookean models were satisfactory up to $50 \%$ of the strain rate. Beyond this point, these models considerably underestimated the behavior of the material.

- The Yeoh model predictions matched the experimental test data exactly, although a small error occurred at small deformations.

The Yeoh model was therefore chosen to describe the hyperelastic behavior of tire rubber.

\subsection{Interactions}

\subsubsection{Tire/rim contact}

The contact of the tire with the rim was simplified by making the assumption that the tire sticks to the rim.

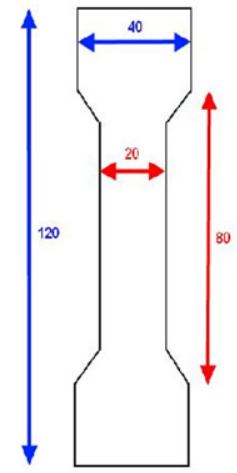

en $(\mathrm{mm})$

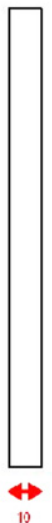

Zone "Carcass"
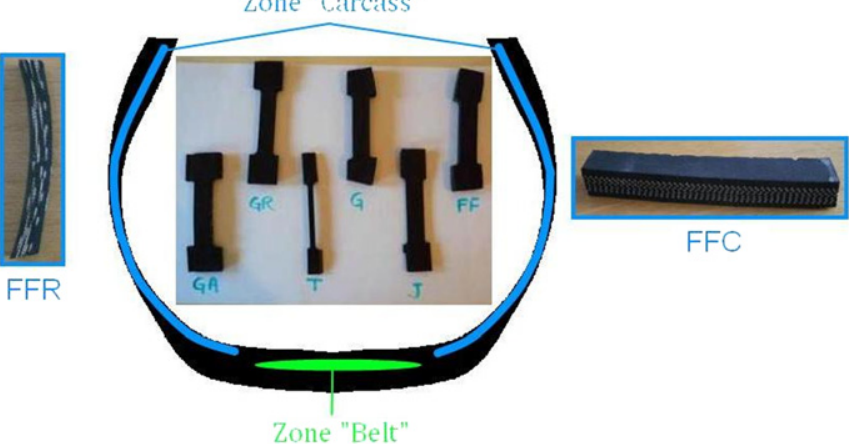

FFC

Fig. 6. Samples: CE stands for the belt and FF for the carcass. C and R (circumferential or radial) denote the direction in which the sample was cut.

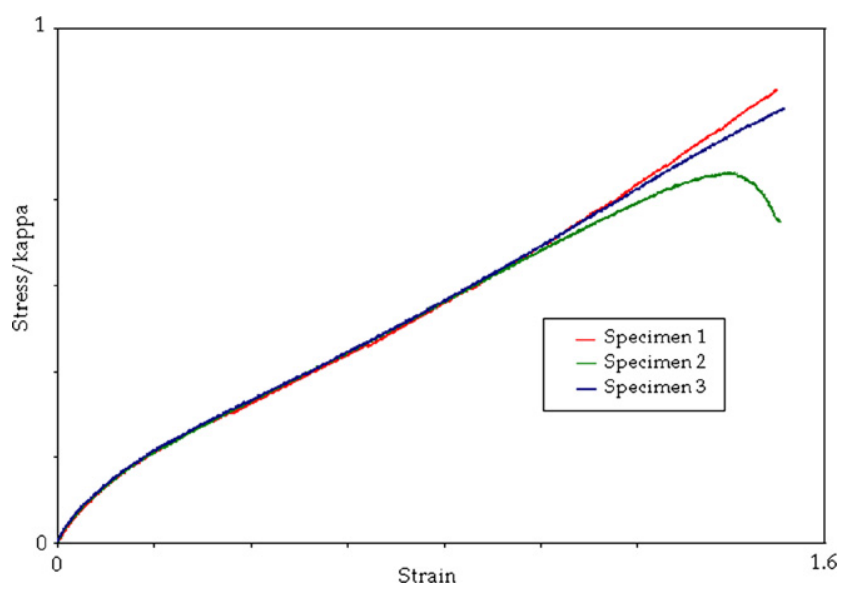

Fig. 7. Stress-strain curve obtained on tread rubber.

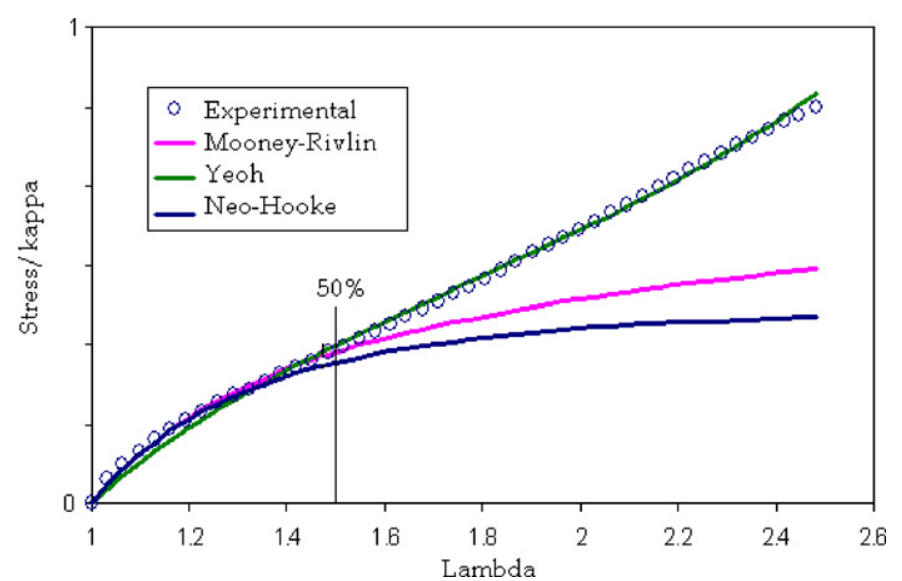

Fig. 8. Behavior of rubber. 


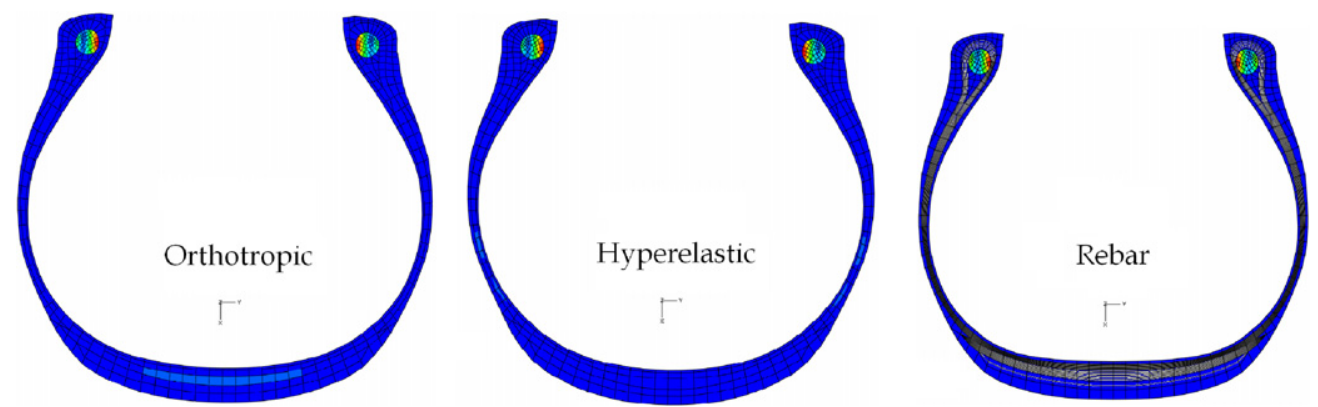

Fig. 9. 2-D axisymmetric finite element model.

\subsubsection{Coulomb friction at tire/ground contact}

The tire/ground contact complicates the finite element model, since contact and friction problems are highly non-linear. However, these effects cannot be ignored. The contact problem was described in the FEM model using a "soft" contact approach with exponential regularization [1]. The Coulomb friction was modeled using a stiffness method. The stiffness method used for friction in ABAQUS/Standard is a penalty method that permits some relative motion of the surfaces [2].

\section{Finite element analysis}

All the finite element calculations were carried out using ABAQUS/Standard Version 9-EF on an HP server with two Xeon quadcore processors cadenced at $3 \mathrm{GHz}$, using the parallel code version.

\subsection{Static analysis}

The first step in studying the tire's behavior consisted in performing a 2-D inflation analysis. The second step consisted in determining the footprint, which is the static deformed shape of the pressurized tire produced by a vertical dead load (corresponding to the weight of an airplane). A three-dimensional model is required for this analysis. The finite element mesh used with this model is obtained by revolving the axisymmetric cross-section about the axle of the rim.

\subsubsection{Inflation analysis}

Inflation analysis (see Fig. 9) is mainly performed on the axisymmetric model in order to check the integrity of the model, to determine the deformed shape of the inflated tire, to locate the stresses in the carcass, belts and rubber components and to set the basis for the 3-D FE model. The group element CGAX4 $(\mathrm{H})$ and $\mathrm{CGAX} 3(\mathrm{H})$ from the ABAQUS element library was selected to represent all the rubber parts. These are four- and three-nodded elements with twist and constant pressure accounting for the incompressibility of the rubber material. The reinforcement materials were modeled in three ways:

- with CGAX4(H) and CGAX3(H) elements for orthotropic elastic model;

- with CGAX4(H) and CGAX3(H) elements for isotropic hyperelastic model;

- with SFMGAX1 for Rebar elements (two-nodded linear axisymmetric surface element with twist).

The three models inflated at the nominal pressure are presented in Fig. 9. The deformation of the belt zone given by the orthotropic and hyperelastic models does not correspond to the physical shape of the inflated tire as the Rebar model does.

\subsubsection{Analysis of a vertically loaded tire}

The inflation analysis of the axisymmetric model was followed by a static 3-D analysis of a vertically loaded tire. This analysis consisted of three steps:

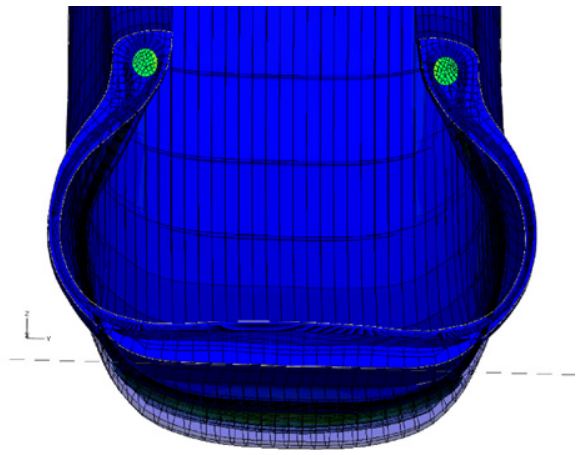

Fig. 10. Loaded and unloaded half-tire geometry given by the Rebar model.

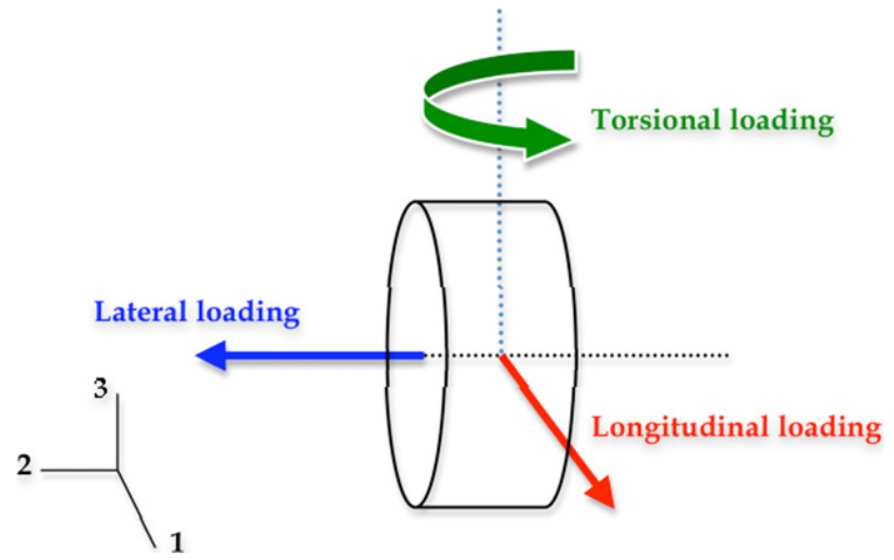

Fig. 11. Lateral, longitudinal and torsional loadings.

- In the first step, the 3-D model was created by rotating the axisymmetric model around the tire axis.

- In the second step, the 3-D tire was inflated and the ground surface was gradually moved towards the tire axis until a prescribed ground displacement value was reached.

- In the third step, the prescribed ground displacement value was replaced by a constant force acting as the airplane weight. This step served as the basis for the subsequent quasi-static and/or dynamic analyses. The results of the vertically loaded tire analysis are shown in Fig. 10.

\subsection{Quasi-static analysis}

In the first validation step, the numerical vertical and horizontal deflections and the footprint shape and area were compared with the manufacturer's data.

Next, three types of loading simulations were performed in order to compare the lateral, longitudinal and torsional stiffnesses obtained with the three models tested with the manufacturer's data (see Fig. 11). 


\section{Results}

Simulations were performed under static vertical loads $F_{z}$ ranging from 0.125 to 0.75 of $f_{0}$. The inflation pressure $p_{0}$ was 17.2 bars and the static friction coefficient $\mu_{s}$ was 0.65 . $p_{0}$ and $f_{0}$ stand for the references values of the pressure and the vertical load respectively. The orthotropic and hyperelastic models involve about 100000 degrees of freedom (DOF), while the Rebar model has about 210000 DOF. Total run time for a complete analysis, including inflation, footprint, lateral, longitudinal and torsional analyses was about 6000 seconds in the case of the most complex Rebar model.

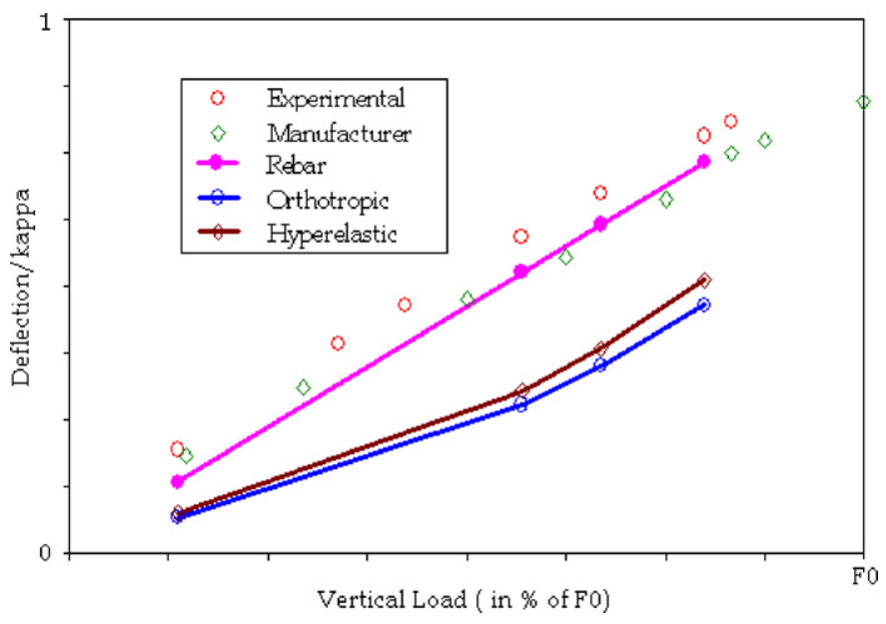

Fig. 12. Load/deflection curves.

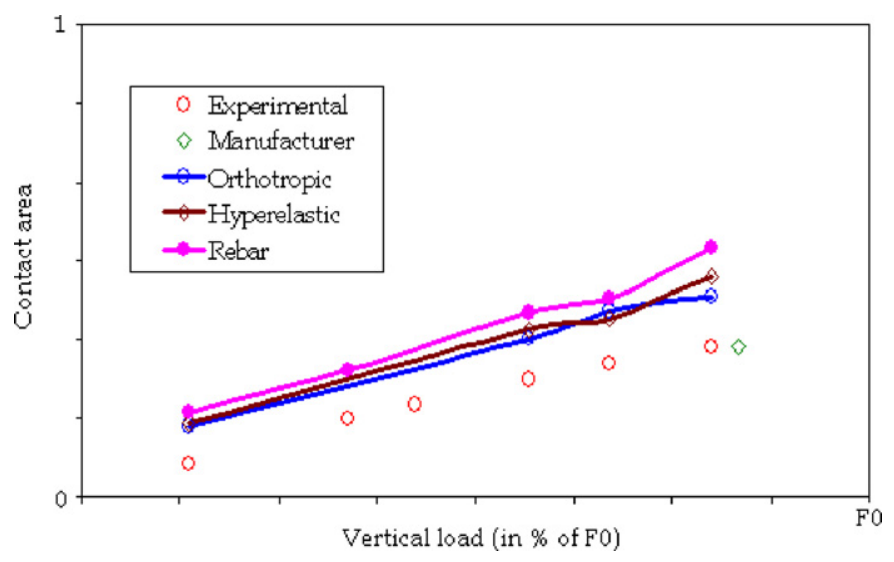

Fig. 13. Contact area curves.

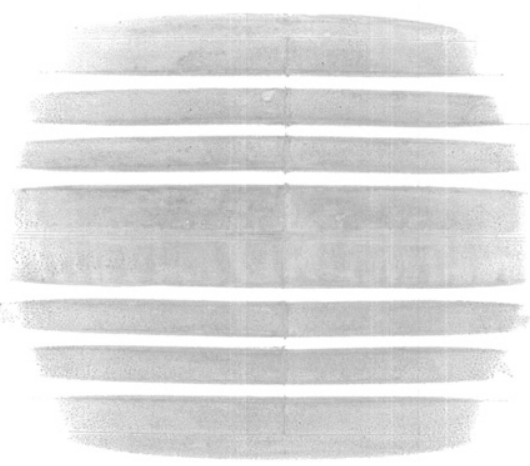

(a) Experimental

\subsection{Static results}

\subsubsection{Load-deflection curves}

Fig. 12 shows the load-deflection curves obtained with the three material models, in comparison with the manufacturer's data and the experimental results.

It can be seen from these comparisons that there was a good agreement between the reference data (manufacturer's data and experimental results) and the values obtained with the (R) model. However, the results obtained with the $(\mathrm{H})$ and $(\mathrm{O})$ models differed considerably from the reference data. This means that the $(\mathrm{H})$ and (O) models are much stiffer than the real tire stiffness measured in the vertical loading direction.

\subsubsection{Contact area}

Fig. 13 gives the contact area vs. loading curves obtained with the three tire models, in comparison with the manufacturer's data and the experimental results. These comparisons show that, with all 3 models $(\mathrm{H}, \mathrm{O}, \mathrm{R})$, the contact areas predicted were larger than those corresponding to the experimental and manufacturer's data, mainly because we did not take the tire grooves into account. The contact area was about 35\% larger without the grooves.

The next two figures (Figs. 14 and 15) give the evolution of the contact area depending on the loading force and the shape of the contact area predicted using a grooved model.

As we can see here, the contact area could only be predicted by modeling a grooved tire.

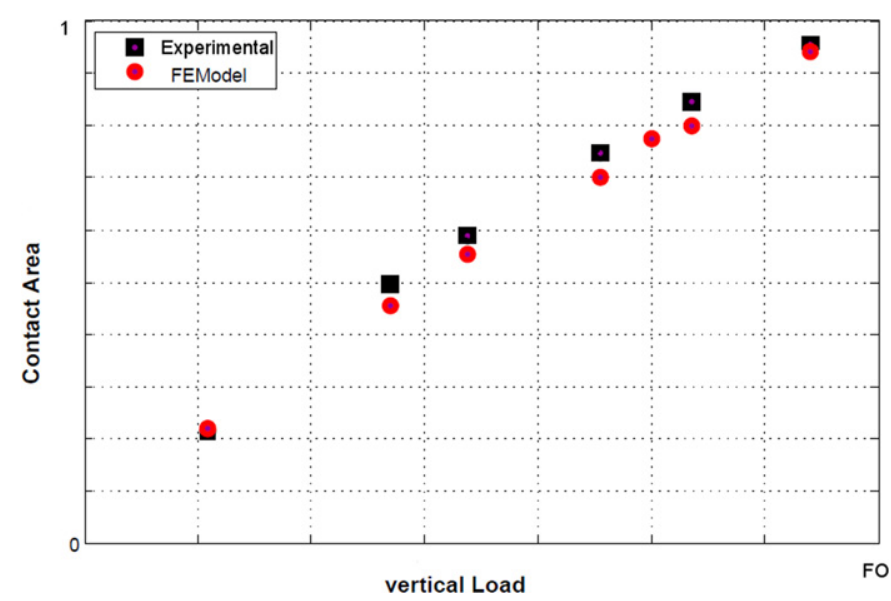

Fig. 14. Evolution of the contact area in the case of a grooved tire.

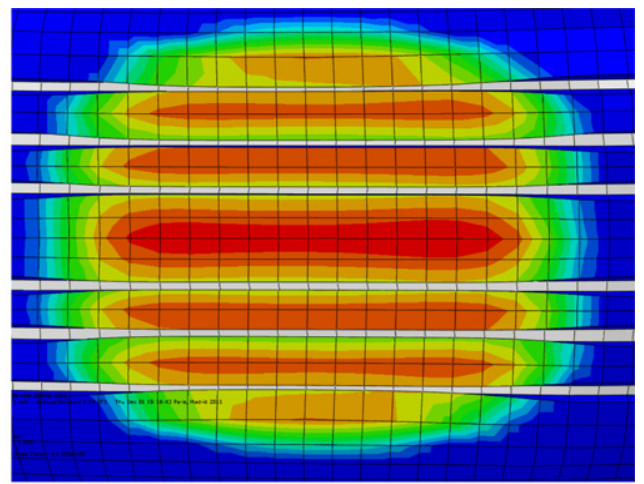

(b) Numerical

Fig. 15. Experimental and numerical footprints at $0.44 f_{0}$. 
Table 1

Changes in sidewall geometry during inflation.

\begin{tabular}{lllll}
\hline & $\begin{array}{l}\text { Inflation } \\
\text { pressure }\end{array}$ & $\begin{array}{l}\text { Left } \\
\text { sidewall }\end{array}$ & $\begin{array}{l}\text { Tire } \\
\text { tread }\end{array}$ & $\begin{array}{l}\text { Right } \\
\text { sidewall }\end{array}$ \\
\hline Experimental & $p_{0}$ & 1 & 1 & 1 \\
Orthotropic model & $p_{0}$ & 0.98 & 9.48 & 0.98 \\
Hyperelastic model & $p_{0}$ & 0.97 & 9.59 & 0.97 \\
Rebar model & $p_{0}$ & 0.89 & 1.08 & 0.89 \\
\hline
\end{tabular}

Table 2

Lateral deflection.

\begin{tabular}{lll}
\hline & Vertical load & Lateral deflection \\
\hline Experimental & $f_{1}$ & 1 \\
Orthotropic model & $f_{1}$ & 0.87 \\
Hyperelastic model & $f_{1}$ & 0.89 \\
Rebar model & $f_{1}$ & 0.95 \\
\hline
\end{tabular}

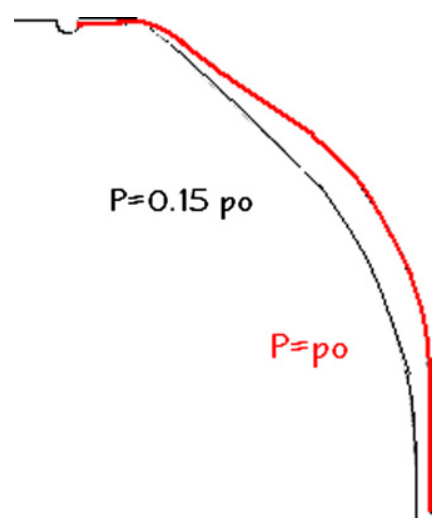

Fig. 16. Sidewall profiles for $0.15 p_{0}$ and $p_{0}$.

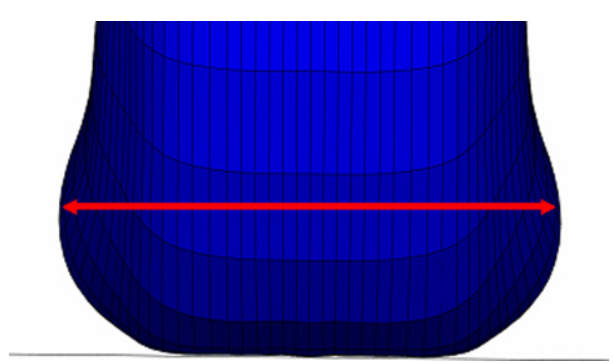

Fig. 17. Lateral deflection at $f_{1}$.

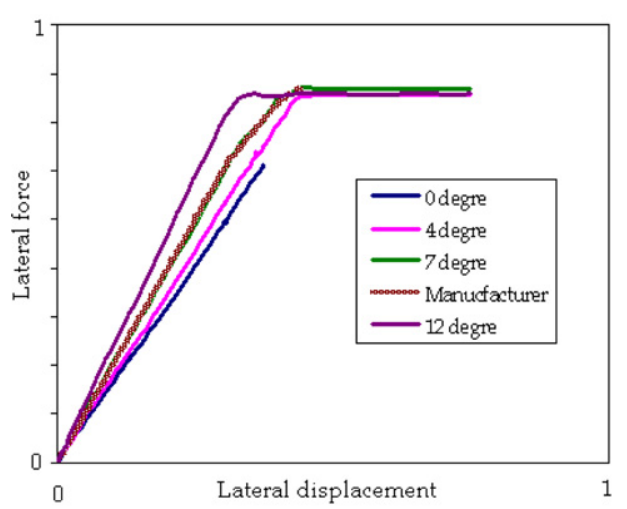

(a)
4.1.3. Changes in the tire sidewall deflection during inflation and loading

These experimental tests were conducted with several inflation pressures and vertical loads.

In Table 1 comparisons are made between the experimental data and the three models tested, in terms of the changes in the sidewall geometry at a pressure $p_{0}$ and a vertical load $F_{z}=0.585 f_{0}$ (see Fig. 16).

Table 2 presents the lateral deflection of the tire measured at the extreme points of the sidewall (Fig. 17) during vertical loading with a force $f_{1}=0.585 f_{0}$.

These comparisons show that the Rebar model gives the most accurate aircraft tire predictions.

In the next section, we will present the results obtained using the Rebar model alone. As explained above, the definition of Rebar elements in shells and surface elements is based on three geometric properties: the cross-sectional area $(A)$ of each individual Rebar element, the spacing $(S)$ between the elements and their orientation $(\theta)$ with respect to the local coordinate system. Since, the properties of Rebar elements depend on the elastic modulus $(E)$, we focused here on the effects of these geometrical and material properties on the lateral, longitudinal and torsional responses of the tires simulated.

\subsection{Geometrical effects}

\subsubsection{Carcass orientation effects}

Four carcass angles $\theta\left(0^{\circ}, \pm 4^{\circ}, \pm 7^{\circ}\right.$ and $\left.\pm 12^{\circ}\right)$ were selected as design variables for investigating the effects of the carcass angle on the tire's lateral, longitudinal and torsional stiffness. This sensitivity study was performed to determine the exact orientation of the fibers in the tire because these orientations cannot be measured directly.

Fig. 18(a) gives the lateral force as a function of the lateral displacement. In this figure, the slope gives the lateral stiffness $k_{Y}$ of the tire (the force is applied to the center of the rim). It can be seen from this figure that the stiffness increases with $\theta$.

Fig. 18(b) gives the longitudinal force as a function of the longitudinal displacement. In this figure, the slope gives the longitudinal stiffness $k_{X}$ of the tire. Regardless of the orientation, the stiffness predicted by the model was always greater than the manufacturer.

Fig. 19 gives the torsional moment as a function of the rotational displacement. In this figure, the curves give the torsional stiffness $k_{M_{z}}$ of the tire. No strong dependence on $\theta$ can be observed, and the values predicted were always greater than the manufacturer's.

The values $\theta= \pm 7^{\circ}$ gave the most accurate lateral behavior $k_{Y}$.

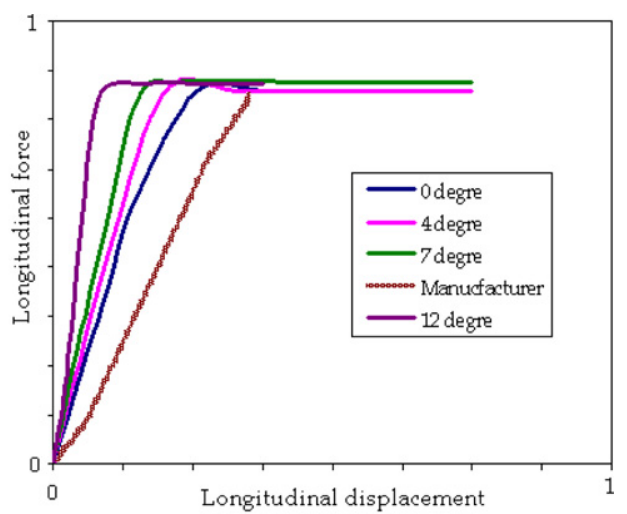

(b)

Fig. 18. Effects of $\theta$ on $k_{Y}$ and $k_{X}$ tire stiffness. 


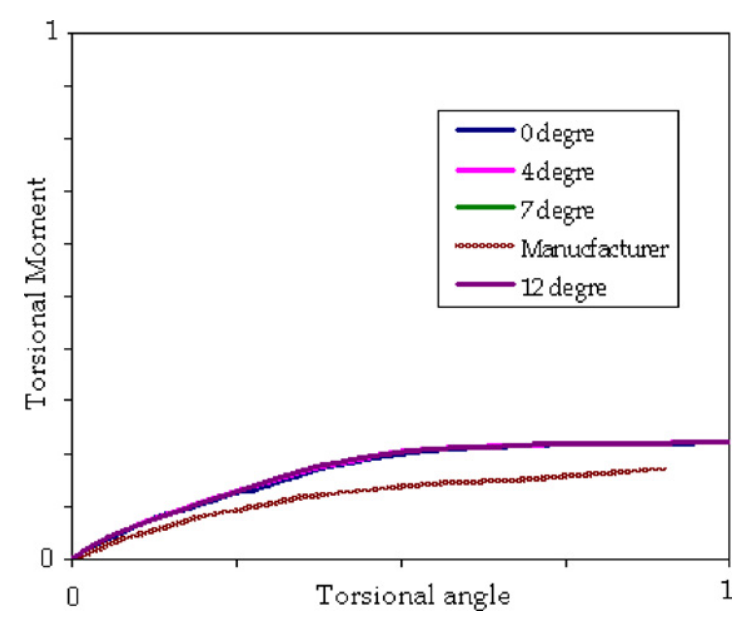

Fig. 19. $\theta$ effects on $k_{M_{z}}$ tire stiffness.

\subsubsection{Belt orientation effects}

Three belt angles, $\kappa= \pm 70^{\circ}, \pm 80^{\circ}$ and $90^{\circ}$, were selected. The carcass angle $\theta$ was kept equal to $\pm 7^{\circ}$.

Fig. 20 shows $k_{Y}$ and $k_{X}$ at each angle $\kappa$. When $\kappa$ differed from $90^{\circ}, k_{Y}$ was constant and stiffer. $k_{X}$ was slightly less stiff, while $k_{M_{Z}}$ seemed to be insensitive to the orientation of the belt.

This finding means that woven belt cords increase the lateral stiffness of tires, but decrease the longitudinal stiffness. In fact, at $\kappa=90^{\circ}$, belt cords have no effect in the lateral direction as the fiber orientation is orthogonal to the loading direction. However, in the case of woven cords, the belt rigidity $E_{\text {belt }}$ also contributes to the stiffening in the lateral direction and to the softening in the longitudinal direction.

\subsubsection{Rebar spacing effects}

Four fiber spacing values $\left(S_{1}=S / 2, S_{2}=S, S_{3}=4 S\right.$ and $S_{4}=$ $10 S$ ) were selected for investigating the effects of $S$ on the tire's lateral, longitudinal and torsional stiffness. $S$ denotes the distance measured between two fibers in the reinforced layers. $\theta$ was assumed to be always equal to $\pm 7^{\circ}$.

Figs. 21 and 22 give the $k_{Y}, k_{X}$ and $k_{M_{Z}}$ stiffness depending on the fiber spacing. As was to be expected, increasing the fiber spacing $S$ decreased the stiffness $k_{Y}$ and $k_{X}$. However, $k_{M_{Z}}$ remained constant at all the spacing values tested.

\subsection{Effects of the material used}

Five materials are mainly used in the field of tire manufacture these days: rayon, nylon, polyester, aramid, and steel [19]. Because of their high modulus, steel and aramid are mostly used in the radial belts and single-ply carcasses of large radial tires. Rayon is used in both the carcasses and belts of passenger cars radial tires, but is less suitable for heavy-duty tires. Modern polyester cord is an excellent carcass textile, which is used along with steel belts in passenger cars and light trucks tires and is becoming the most widely used material for this purpose worldwide. However, it lacks the toughness and heat resistance required for large tires. Nylon is the most suitable textile for large bias truck, earthmover and aircraft tires, but nylon like polyester, is not stiff enough for radial belts.

Some sensitivity studies were performed on fiber moduli. Six belt fiber moduli $E_{\text {belt }}=\left(E_{\text {steel }}\right.$ or $E_{\text {rayon }}$ or $E_{\text {kvelar }}$ or $E_{\text {polyester }}$ or $E_{\text {nylon }}$ or $\left.E_{\text {nylon }} / 10\right)$ and two carcass fiber moduli $E_{c}=\left(E_{\text {polyester }}\right.$ or $\left.E_{\text {nylon }}\right)$ were selected as the design variables for investigating the effects of the material on the tire response. $E_{b e l t}$ and $E_{c}$ denote the reference elastic moduli in the belt and carcass layers, respectively. $\theta$ is assumed to be equal to $\pm 7^{\circ}$.

Figs. 23 and 24 show the predicted $k_{Y}, k_{X}$ and $k_{M_{Z}}$ stiffness corresponding to each modulus ( $E_{\text {belt }}$ and $\left.E_{\text {carcass }}\right)$. Since $E_{\text {carcass }}$ was always greater than $E_{n y l o n}$, no significant changes in $k_{Y}$ and $k_{X}$ were observed. The predicted values of $k_{X}$ were always greater than the manufacturer's values. When $E_{\text {carcass }}$ was beyond this range, $k_{Y}$ decreased slightly and $k_{X}$ decreased significantly. The $k_{X}$

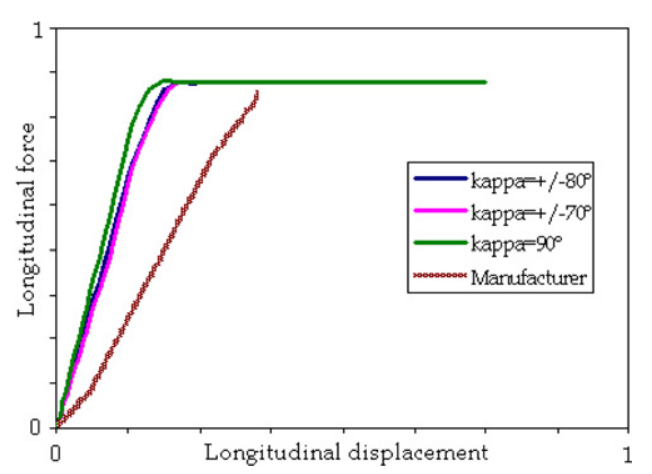

Fig. 20. Effects of $\kappa$ on $k_{Y}$ and $k_{X}$ tire stiffness.
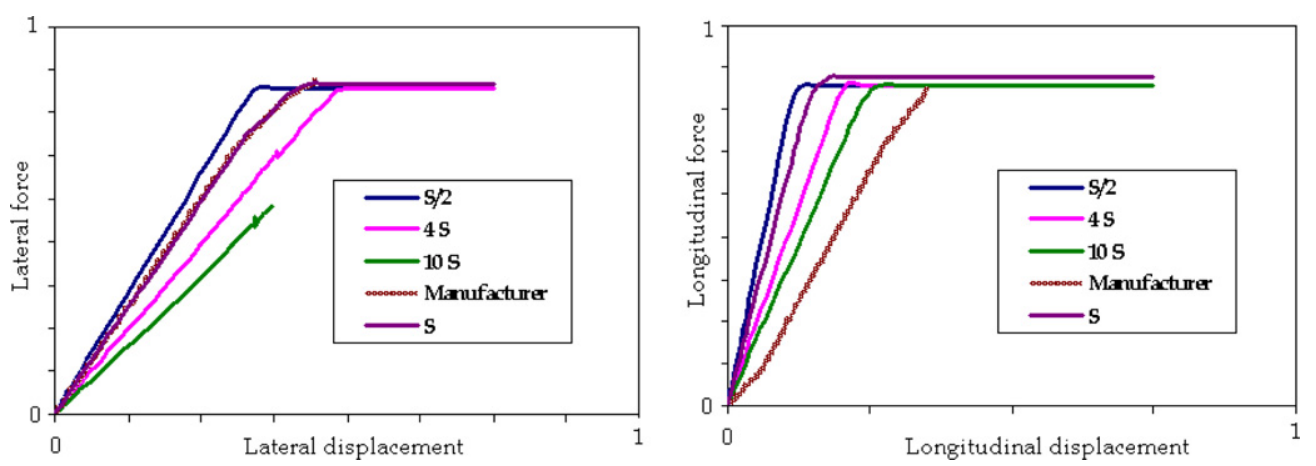

Fig. 21. Effects of $S$ on $k_{Y}$ and $k_{X}$ tire stiffness. 


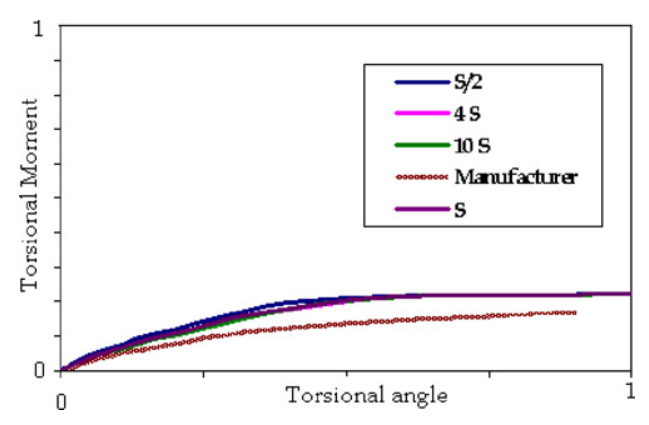

Fig. 22. $S$ effects on $k_{M_{z}}$ tire stiffness.
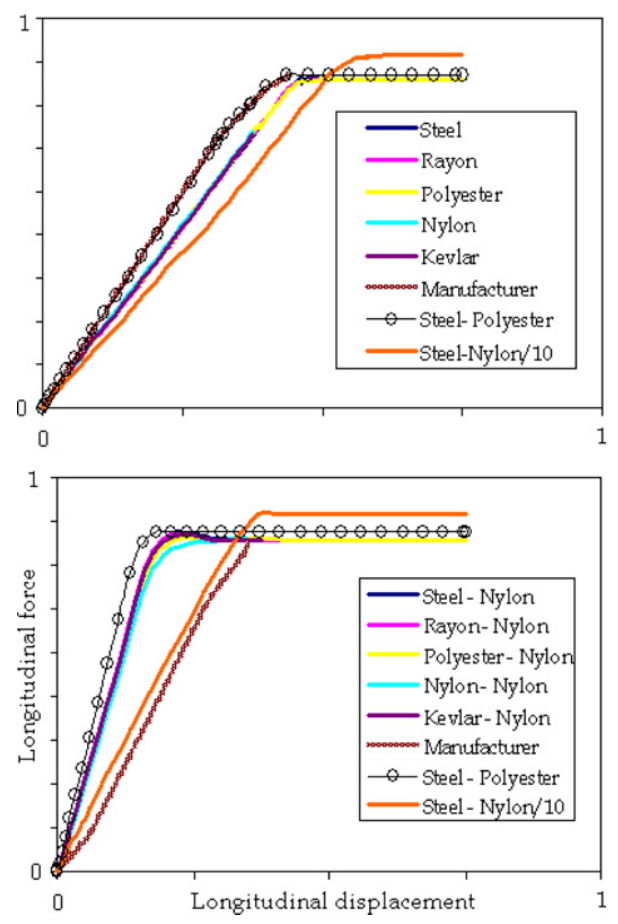

Fig. 23. Effects of the fiber modulus on $k_{Y}$ and $k_{X}$ tire stiffness.

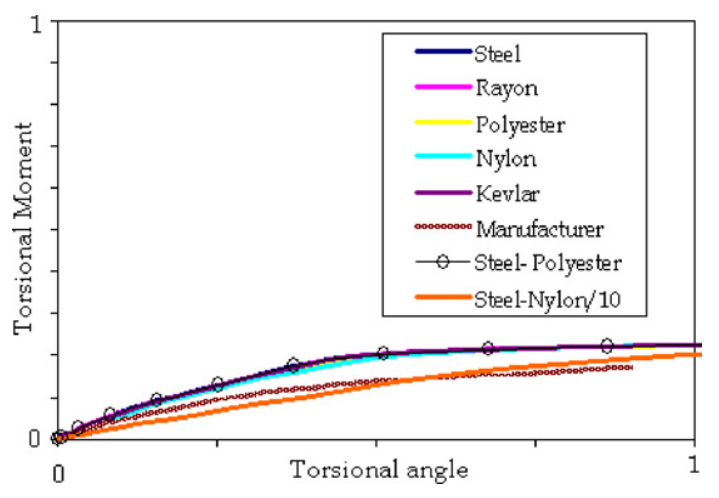

Fig. 24. Effects of the fiber on the tire stiffness $k_{M_{z}}$.

predicted by the Rebar model was therefore very similar to the value specified by the manufacturer. The steel-polyester material showed the greatest lateral stiffness (see Fig. 23).

The tire response seems to be more sensitive to the carcass moduli. Most carcass layers of aircraft tires are presumably made of polyester, as stated in [19].
These sensitivity studies show that the $k_{Y}$ depends on the modulus of the carcass $E_{\text {carcass }}$ while $k_{X}$ depends on $E_{\text {belt }} . k_{Y}$ and $k_{X}$ seem to be strongly coupled. Although many configurations and simulations were performed, it is difficult, however, to determine exactly how are correlated the lateral and longitudinal stiffness.

\section{Conclusion}

In this paper is presented a method for modeling an aircraft tire under severe operating conditions using finite element tools. The Rebar model proved to be the most accurate mean of modeling tire behavior under static and quasi-static loading conditions with a view to dynamic simulations. The Rebar modeling of the reinforced zones also gave the most realistic results. After the many sensitivity studies performed here, the next challenge is to elucidate the strong coupling which seems to exist between the lateral and longitudinal stiffness.

\section{References}

[1] ABAQUS/Standard, Theory Manual and Example Problems Manual, Release 6.9, 2010.

[2] ABAQUS/Standard, Abaqus Analysis User'S Manual, Release 6.9, 2010.

[3] S.K. Clark, R.N. Dogde, Heat generation in aircraft tires under free rolling conditions, NASA contractor report 3629, 1982.

[4] M.H. Ghoreish Reza, Finite element analysis of steel-belted radial tyre with tread pattern under contact load, Iranian Polymer Journal 15 (8) (2006) 667674.

[5] R.M. Jones, Mechanics of Composite Materials, 2nd edition, Taylor \& Francis, 1999.

[6] A. Kongo Kondé, I. Rosu, F. Lebon, O. Brardo, B. Devésa, Etude du comportement en roulement d'un pneu d'avion, Colloque National de Calcul de Structures, Giens 1 (2009) 699-704 (in French).

[7] A. Kongo Kondé, Modélisation du roulement d'un pneumatique d'avion, $\mathrm{PhD}$ thesis, Aix-Marseille University, 2011, p. 232 (in French).

[8] N. Korunovic, M. Trajanovic, M. Stojkovic, Finite element model for steady state rolling tire analysis, Journal of the Serbian Society for Computational Mechanics 1 (1) (2007) 63-79.

[9] J. Lacombe, Tire model for simulations of vehicle motion on high and low friction road surfaces, in: Proceedings of the Winter 2000 Simulation Conference, vol. 1, Hanover, 2000, pp. 1025-1034.

[10] N. Lahellec, F. Mazerolle, J.C. Michel, Second-order estimate of the macroscopic behaviour of periodic hyperelastic composites: theory and experimental validation, Journal of the Mechanics and Physics of Solids 52 (2004) 27-49.

[11] J.L. McCarthy, J.A. Tanner, Temperature distribution in a aircraft tire at low ground speed, NASA technical paper 2195, 1983.

[12] Michelin dans le Benelux, Performances de haut niveau pour le décollage et l'atterrissage, http://www.michelin.be (in French).

[13] K.V. Narasimha Rao, R. Krishna Kumar, P.C. Bohara, R. Mukhopadhyay, A finite element algorithm for the prediction of steady-state temperatures of rolling tires, Tire Science and Technology, TSTCA 34 (3) (2006) 195-214.

[14] J.P. Navarro, Contribution la modélisation du pneumatique de l'Avion, Thèse, Université Toulouse III-Paul Sabatier, 2003 (in French).

[15] O.A. Olatunbosum, E.O. Bolarinwa, Finite element simulation of the tyre burst test, in: Proceedings of the Institution of Mechanical Engineers, vol. 218, 2006 pp. 1251-1258.

[16] J.A. Tanner, R.C. Dreher, S.M. Strubb, E.G. Smith, Tire tread temperatures during antiskid braking and "cornering" on a dry runway, NASA technical paper 2009, 1982

[17] W.D. Versteden, Improving a tire for motorcycle simulation, Thesis, Eindhoven University of Technology, 2005.

[18] J.D. Walter, H.P. Patel, Approximate expressions for the elastic constants of cord-rubber laminates, Rubber Chemistry and Technology 52 (1979) 710-724.

[19] J.D. Walter, A.N. Gent, The pneumatic tire, National Highway Traffic Safety Administration, US Department of Transportation, Washington DC 20590, DOT Contract DTNH22-02-P-07210, August 2005.

[20] G. Yanjin, Z. Guoqun, C. Gang, Influence of belt cord angle on radial tire under different rolling states, Journal of Reinforced Plastics and Composites 25 (10) (2006) 1059-1077. 